\title{
Involuntary Memory and the Development of Retrieval Skills in Young Children
}

\author{
Catherine Sophian and John William Hagen \\ Univervity of Michigan
}

\begin{abstract}
An incidental memory paradigm was used to study involuntary encoding processes and voluntary retrieval strategies in children's memory. Preschool (mean age: 4 years, 4 months) and kindergarten (mean age: 5 years, 10 months) children sorted pictures according to their color or category membership, and then received either a recall test (Experiment 1) or a recognition test (Experiment 2). Better retention of category- than color-sorted items was observed for kindergarten children in free recall, preschool and kindergarten children in cued recall, and neither group in recognition. These results were interpreted in terms of the retrieval strategies used by children in each of the memory tasks. The importance of distinguishing between voluntary and involuntary memory processes, and between acquisition and retrieval, in studies of depth-of-processing was emphasized. Developmental differences in performance appear to derive primarily from the role of voluntary search strategies in retrieval, rather than from age differences in involuntary encoding processes.
\end{abstract}

An important distinction for the study of memory development is one between voluntary and involuntary memory. The memory of the preschool child has been characterized as automatic (Myers \& Perlmutter, in press), nonstrategic (Appel, Cooper, McCarrel, Sims-Knight, Yussen, \& Flavell, 1972; Flavell, 1970; Flavell, Friedrichs, \& Hoyt, 1970), and involuntary (Smirnov, 1973; Smirnov \& Zinchenko, 1969; Yendovitskaya, 1971); and developmental improvements in memory performance have been attributed to the acquisition of voluntary mnemonic strategies (Brown, 1975; Hagen, Jongeward, \& Kail, 1975; Keeney, Cannizzo, \& Flavell, 1967). However, despite this strategic deficit, young children do display impressive memory performance on recognition (Perlmutter \& Myers, 1976), reconstruction (Brown \& Murphy, 1975), and delayed response (Loughlin \& Daehler, 1973) tasks. Moreover, developmental improvements have been found in the preschool years which do not appear to be attributable to increasing strategy utilization (Myers \& Perlmutter, in press). Thus,

This research was supported in part by a USPHS traineeship under Grant \# 5T01 HD 00149 and by the Department of Psychology at the University of Michigan. We would like to thank Marion Perlmutter and Henry Wellman for their helpful comments on previous drafts of this manuscript. Requests for reprints should be sent to: Catherine Sophian, Developmental Psychology Program, University of Michigan, Ann Arbor, MI 48109. 
consideration of nonstrategic aspects of the memory system appears to be necessary to account for early stages of memory development.

The incidental memory paradigm provides a means of investigating children's memory in the absence of strategic encoding processes. The experimenter, through the choice of appropriate orienting tasks, can control the way in which stimulus materials are processed at presentation. This paradigm has become increasingly prominent in the adult literature with the advent of depth-of-processing theory (Craik \& Lockhart, 1972), which postulates that retention is a function of the depth to which materials are processed at encoding. Depth-of-processing research with adults has consistently obtained better memory performance following semantic orienting tasks than following tasks based on formal characteristics of the material (Hyde \& Jenkins, 1973; Craik \& Tulving, 1975). There is also evidence for the importance of comprehension in children's memory for incidentally presented materials. Murphy and Brown (1975) tested fouryear-olds' recall of a set of categorized pictures following five different orienting tasks, and found better retention following tasks which required comprehension than following formal orienting tasks.

Two questions remain unanswered in recent studies of depth-ofprocessing, which are addressed in the present research. The first concerns possible developmental changes in the relative effectiveness of different orienting tasks for subsequent retention; the second concerns the role of retrieval factors in children's performance on incidental memory tasks.

No developmental changes in the relative effectiveness of formal and semantic orienting tasks were found in one study of incidental memory in children from first to fifth grade (Geis \& Hall, 1976); retention was always best following the semantic task. However, it could be argued that the formal tasks used in that study were not the most likely ones to produce a relative advantage for younger children; they were acoustic and orthographic tasks, depending heavily upon verbal knowledge which children in the age range tested are still in the process of acquiring.

In the present study, a formal orienting task based on the colors of pictures was compared with a semantic task based on the category membership of the objects depicted. Preschool and kindergarten children were asked to sort pictures into groups according to color, and according to category; sorting times were recorded as a rough index of the depth of processing required to perform each task. The two tasks were as equivalent as possible: both provided an organizational structure for the items differing only in the features upon which that structure was based. There is evidence that young children prefer to organize pictures on the basis of color, while older children are more likely to rely on category information (Wingard \& Glieberman, Note 2). Developmental changes in the relative effectiveness of color- and category-sorting tasks for involuntary memory might be expected to correspond to these changes in organizational 
preferences. If so, the color-sorting task should produce better retention relative to the category-sorting task, for younger children than for older children.

The retrieval phase of task performance has received little attention in rescarch on incidental memory. While encoding strategies are precluded through the use of an incidental orienting task at presentation, the retrieval task is still a voluntary one in which strategic behavior may play an important role. Consideration of retrieval components of the incidental memory task suggests that differences in memory performance following various orienting tasks may be due to differences in the accessibility, rather than availability, of information in memory (Tulving \& Pearlstone, 1966). That is, differences may arise, not because children store more information in the course of carrying out one orienting task than the other, but because the stored information can be retrieved more readily following one of the tasks. In particular, the accessibility of memory information might be expected to vary as a function of the compatibility of the organization produced at encoding with the retrieval scheme used by the child.

The role of retrieval processes in incidental memory performance may be investigated by examining performance on memory tasks which vary in their retrieval requirements. Free recall performance depends heavily on children's ability to access stored information, and thus should be maximally affected by retrieval processes. Cueing provides a way of facilitating access to stored information, so that differences in the accessibility of memory information should have less effect on cued recall performance. Recognition provides an assessment of memory in which retrieval factors are at a minimum. In Experiment 1, children's memory following formal and semantic orienting tasks was tested with free and cued recall. In Experiment 2, the same types of incidental orienting tasks were followed by a recognition test. The implications of the results of both studies are considered following presentation of the results of each.

\section{EXPERIMENT 1}

The purpose of the first experiment was to determine whether depth-ofprocessing effects in recall are subject to developmental changes in early childhood and to differentiate between differences in accessibility and availability of information in memory. By facilitating access to stored information, cues should reduce the effects of differences in accessibility upon recall performance. However, the effectiveness of retrieval cues in facilitating access to stored information may depend upon the way in which that information is encoded. According to the encoding specificity hypothesis (Tulving \& Thomson, 1973), cues should be effective only to the extent that they match the stored trace of the stimulus. This principle suggests that color cues should be more effective than category cues for retrieval of 
color-sorted items; while category cues should be most effective for retrieval of items which were sorted by category. Some support for this prediction was obtained in the Murphy and Brown (1975) study, in which category cues facilitated performance relative to free recall only in the condition in which a categorization task was performed at presentation. However, that study included only half of the complete design needed to test the encoding specificity hypothesis, since no cues corresponding to the formal orienting tasks were provided. In the present study, orthogonal color and category cues were used to test recall following both color- and category-sorting tasks.

\section{Method}

Subjects. Sixteen children from each of two age groups were tested. The preschool children were between 3 years, 7 months and 4 years, 10 months of age (mean age: 4 years, 4 months); the kindergarten children were between 5 years, 5 months and 6 years, 9 months of age (mean age: 5 years, 10 months). There were approximately equal numbers of boys and girls in each age group. Children were tested individually at their schools by a female experimenter. The testing session lasted about $15 \mathrm{~min}$.

Materials. The stimuli consisted of colored pictures of six familiar objects from each of three categories: clothes, furniture, and vehicles. The pictures were line-drawings on $3 \frac{3}{4} \times 41 / 2$ inch cards; two pictures from each category were red, yellow, and blue, respectively. The pictures were divided into two sets such that each set contained one picture of each color from each category.

Procedure. The children were taken from their classrooms to an unoccupied room in the school for testing. The task was introduced as a "picture game;" no mention was made of any memory requirement. The experimenter presented the first set of pictures to the child, asking him to name each picture as it was presented. The pictures were placed successively into a $3 \times 3$ matrix, with no two pictures in any row or column of the same color or from the same category. The child was then asked to sort the pictures into groups on the basis of their color or category membership. If he had trouble carrying out the sorting instructions, the experimenter prompted him by asking him to find all the items for one group at a time (e.g., all the red pictures, or all the clothes). The child was asked to correct any errors he made. A stopwatch was used to measure sorting times from the moment the child touched the first picture until the last one had been correctly sorted.

The second sorting task was presented immediately after the first. The procedure was exactly the same, except that each child was asked to produce the opposite type of sort from the one he had done previously (color or category). The order of sorts was counterbalanced across subjects, but the two sets of pictures were always presented in the same order. 
The experimenter waited about 1 min after the second sort had been completed before asking for recall. The child was then asked to name all the pictures he could remember from either set. Recall continued with occasional prompts from the experimenter, until the child indicated that he could not remember any more items.

Two cued recall tests were then administered, with the order counterbalanced across subjects: one in which color cues were provided, and one utilizing category cues. In each case, the child was told that "we're going to start over" and "try another way to think of all the pictures" in order to make clear that he should name all the pictures he could think of, and not just the ones he had omitted previously. The three cues of each type were presented in a fixed order, each successive cue being presented when the child indicated that he could not recall any more items to the previous cue.

When the second cued recall test had been completed, the child was praised for his performance, thanked for his participation, and taken back to his classroom.

\section{Results}

Sorting times. The mean number of seconds taken by children in each age group to complete the sorting tasks is presented in Table 1. A 2(sort type) $\times 2$ (age) $\times 2$ (sort order) analysis of variance, with age and sort order as between-subjects variables, indicated that color sorts were completed significantly faster than category sorts $(F[1,28]=43.8, p<.001)$. There were no other significant effects on sorting time.

Free recall. The mean number of items from color- and category-sorted lists produced in free recall by children in each age group may be seen in Fig. 1. A 2(sort type) $\times 2$ (age) $\times 2$ (sort order) analysis of variance, with age and sort order as between-subjects variables, revealed significant main effects of age $(F[1,28]=7.36, p<.025)$ and sort type $(F[1,28]=6.16$, $p<.025)$. As expected, older children recalled more items than younger

TABLE 1

Mean Seconds Required to Complete Sorting Tasks

\begin{tabular}{ccc}
\hline & \multicolumn{2}{c}{ Sort type } \\
\cline { 3 - 3 } & Color & Category \\
\hline Experiment 1 & & \\
Preschool & 24.38 & 45.56 \\
Kindergarten & 18.25 & 42.06 \\
Experiment 2 & & \\
Preschool & 18.56 & 33.56 \\
Kindergarten & 16.31 & 21.25 \\
\hline
\end{tabular}




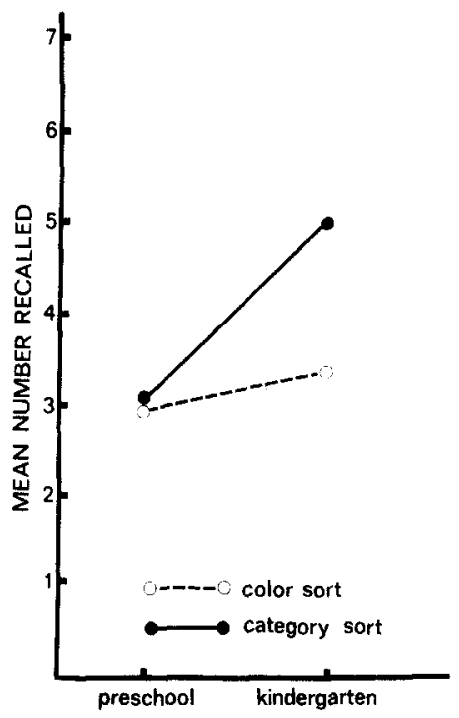

FIG. 1. Mean number of items produced in free recall as a function of age and sorting task $(\operatorname{maximum}=9)$.

children; and more items were recalled from the category-sorted than from the color-sorted lists.

The age $\times$ sort type interaction was marginally significant $(F[1,28]$ $=3.72, p<.10$ ), with older children showing a greater difference in recall of items sorted according to color vs category dimensions than did the younger children. Planned-comparison $t$-tests revealed a reliable difference in recall between the two lists for older children $(t[31]=2.22, p<.05)$ but not for younger children $(t[31]=.25, p>.80)$.

The final effect which reached significance in the free recall analysis was the sort type $\times$ sort order interaction $(F[1,28]=46.8, p<.001)$. This interaction appeared to reflect a recency effect: a greater difference in recall between the two lists was obtained when the color sort preceded the category sort than when the reverse sort order was used.

The presence of systematic differences in sorting time between the two sorting tasks raises the possibility that the observed differences in recall may be due to differences in exposure time rather than the depth of processing induced by the sorting tasks. However, regression analyses performed with sorting time as the predictor and recall scores as the dependent variable produced no significant effects of sorting time, either in an analysis of all the scores combined $\left(F<1 ; r^{2}=.00001\right)$, or in separate regressions for the first and second lists, and younger and older children, performed in order to take into account these additional sources of variance (all $F$ 's $<1$; $r^{2}$ 's $<.05$ ). In contrast, a regression with sort type as the predictor was highly significant $\left(F[1,62]=26.5, p<.001 ; r^{2}=.30\right)$. Hence, it does not 
appear that sorting time differences can account for the effects of sort type on recall.

Cued recall. The results of the cued recall tests are presented in Fig. 2. A 2 (sort type) $\times 2$ (cue type) $\times 2$ (age) $\times 2$ (sort order) $\times 2$ (cue order) analysis of variance, with age, sort order, and cue order as betweensubjects variables, was performed on these data. Again, older children recalled more items than did younger children $(F[1,24]=13.86, p<.005)$, and more items were recalled from the category-sorted than from the colorsorted lists $(F[1,24]=12.78, p<.005)$. Also, a significant sort type $\times$ sort order interaction $(F[1,24]=15.92, p<.001)$ again appeared to reflect a recency effect. However, the age $\times$ sort type interaction did not even approach significance in this analysis $(F<1)$. When cues were provided, both younger and older children recalled more of the categorysorted than the color-sorted pictures.

Category cueing led to higher levels of recall than did color cueing $(F[1,24]=12.78, p<.005)$. The sort type $\times$ cue type interaction was also significant $(F[1,24]=20.95, p<.001)$. The difference between colorand category-cued recall was greater for category-sorted than color-sorted items. Nevertheless, category cues were more effective than color cues even for recall of color-sorted items $(t[31]=5.42, p<.001)$.

Again, regression analyses failed to support the possibility that differences in exposure time might be responsible for the observed effects of sorting task. A regression with sorting time as the predictor did not reveal significant effects on cued recall ( $F<1 ; r^{2}=.002$ ); but sorting task was a reliable predictor of cued recall $\left(F[1,62]=8.05, p<.01 ; r^{2}=.11\right)$.

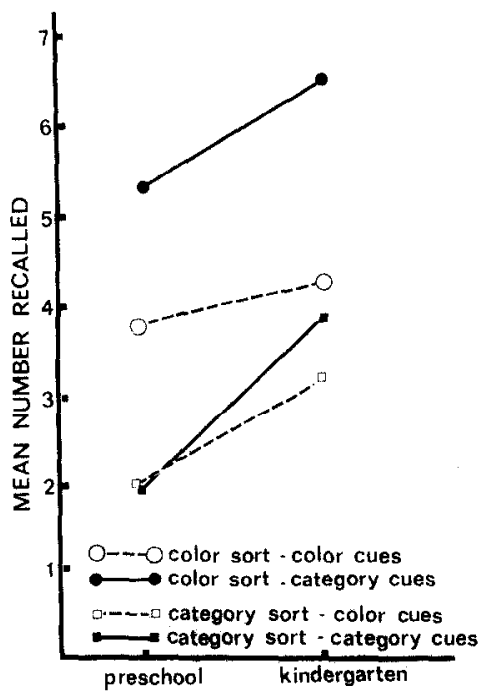

Fig. 2. Mean number of items produced in cued recall as a function of age, sorting task, and type of cue (maximum $=9$ ). 
Clustering. Clustering measures were computed on the free recall data, combining the two lists since they were recalled simultaneously. The RR index of clustering was used, as this measure has been found to be independent of level of recall in simulation studies (Murphy, Puff, \& Campione, Note 1). Clustering scores were calculated on the basis of both category and color dimensions of organization. Children in both age groups showed above-chance levels of category clustering (preschool: $\bar{X}=.49$. $t[15]=4.59, p<.001$; kindergarten: $\bar{X}=.42, t[15]=3.05, p<.01)$, but color clustering was below chance level (preschool: $\bar{X}=.19$ : kindergarten: $\bar{X}=.23$; chance $=.29$ ).

In order to assess possible effects of the incidental tasks on clustering, separate clustering scores were calculated for the two lists, based on the frequency with which items were recalled together which were from the same list and of the same color or category. Mean clustering scores for each list, based on color and category dimensions, may be seen in Table 2. Only the category-based clustering of category-sorted items for the older children was significantly above chance $(t[15]=2.58, p<.05$; chance $=.12)$. A 2 (age) $\times 2$ (sort order) $\times 2$ (sort type) $\times 2$ (clustering dimension) analysis of variance, with age and sort order as between-subjects variables, revealed a significant effect of clustering dimension $(F[1,28]=15.1$, $p<.001)$, and a marginally significant effect of sort type $(F[1,28]=2.97$, $p<.10$ ). Children clustered more on the basis of category than on the basis of color, and tended to show more clustering in recalling items from the category-sorted than the color-sorted lists. A significant sort type $\times$ sort order interaction $(F[1,28]=6.46, p<.025)$ reflected greater clustering of items from the more recently presented list. There was also a significant age $\times$ sort order $\times$ clustering dimension interaction $(F[1,28]$ $=9.37, p<.005$ ). The younger children showed a greater difference between color- and category-based clustering when the category-sorting task preceded the color-sorting task than when the reverse sort order was

\section{TABLE 2}

Mean Scores on RR Clustering Index

\begin{tabular}{lll}
\hline & \multicolumn{2}{c}{ Clustering dimension } \\
\cline { 2 - 3 } Age group & Color & Category \\
\hline Preschool & & .19 \\
$\quad$ Color-sorted items & .04 & .20 \\
Category-sorted items & .10 & \\
Kindergarten & & .09 \\
$\quad$ Color-sorted items & .06 & .28 \\
Category-sorted items & .08 & \\
\hline
\end{tabular}


used; while older children showed a greater difference between the two types of clustering when the color-sorting task occurred first.

\section{EXPERIMENT 2}

In Experiment 2, a recognition task was used in order to assess the effects of sorting task on children's memory when retrieval factors are at a minimum. Effects of sorting task on recognition memory should be attributable to differences in the availability of information in memory, since differences in accessibility would not be expected to affect recognition performance. On the other hand, if no effects of sorting task are observed in recognition, the suggestion would be that differences in accessibility were the primary source of the effects of sorting task on recall found in Experiment 1. Thus, a comparison of recognition data with the free and cued recall results of Experiment 1 should help to clarify the relative importance of storage and retrieval factors in producing effects of depthof-processing on children's memory.

\section{Method}

Subjects. Sixteen preschool children ( 3 years, 7 months to 4 years, 11 months of age; mean age: 4 years, 3 months) and 16 kindergarten children ( 5 years, 7 months to 6 years, 6 months; mean age: 5 years, 11 months) participated in this study. There were approximately equal numbers of boys and girls in each age group. Children were tested individually by a female experimenter in an isolated room in their schools. The testing session lasted about $15 \mathrm{~min}$.

Materials. The stimuli were pictures of familiar objects similar to those used in Experiment 1. They included four colored pictures (red, yellow, blue, or green) from each of four categories (clothes, furniture, toys, and vehicles). Two sets of eight pictures each were used for the sorting tasks. Each set contained only two colors and two categories, so that entirely different colors and categories appeared in each. Within each set, two of the items from each category were one color, and two the other color. Sixteen additional items served as distractors in the recognition test. They consisted of four new items from each category, one in each of the four colors. Thus each distractor item matched one of the presentation sets in color, and one in category.

Procedure. As in Experiment 1 the task was described to the children as a "picture game," with no mention of any memory requirement. The experimenter presented the first set of items, successively placing each picture into a $2 \times 4$ matrix, such that each row contained two instances of each color and category, and no column contained two pictures which were the same in color or category. When all of the pictures had been presented, the child was asked to put them into two piles, so that all of the pictures 
in each pile were the same color or the same category. After the child completed this task, the second set of pictures was presented in the same way, and the opposite type of sort was requested. Children's sorting times were measured with a stopwatch. The order in which the two tasks were presented, and the set of pictures used for each task, were counterbalanced across subjects.

Following completion of the sorting tasks, there was a 2-min delay while the experimenter combined the sorted pictures with the distractors in a fixed random sequence. The entire set of pictures was then presented one at a time for the recognition test. The child was asked to indicate whether each picture was one that had been shown to him before, or a new one. No feedback was given as to the correctness of his responses.

Results

Sorting times. Mean sorting times for Experiment 2 are presented in Table 1. A 2(sort type) $\times 2$ (age) $\times 2$ (sort order) analysis of variance revealed significant effects of age $(F[1,28]=6.24, p<.025)$, sort type $(F[1,24]=19.07, p<.001)$ and age $\times$ sort type $(F[1,28]=4.86, p$ $<.05$ ). Older children sorted the pictures more quickly than younger children; and color sorts were completed faster than category sorts. The difference in time required to complete category and color sorts was greater for the younger than the older children.

Hits. The mean number of sorted items which were correctly recognized as "old" may be seen in Table 3. A 2(sort type) $\times 2$ (age) $\times 2$ (sort order) analysis of variance, with age and sort order as between-subjects variables, revealed only a significant effect of sort type $\times \operatorname{sort}$ order $(F[1,28]=7.26$, $p<.025)$. This effect is similar to those found in Experiment 1 and again appears to reflect recency.

A second analysis on data from the first-sorted list only provides evidence that the absence of age and sorting task differences was not due to ceiling effects. The mean number of hits for items from the first list was 6.47 , significantly less than the mean of 7.44 for the second list ( $t[31]$ $=2.71, p<.01$ ). Therefore, if effects of age and sort type were merely masked by the high level of performance overall, they should emerge in an analysis of first-list data only, where performance is lower. However, a 2 (sort type) $\times 2$ (age) between-subjects analysis of first-list hits failed to

TABLE 3

Mean Number of Hits in Recognition (Maximum $=8$ )

\begin{tabular}{lcc}
\hline Age group & Color sort & Category sort \\
\hline Preschool & 6.81 & 7.13 \\
Kindergarten & 7.13 & 6.75 \\
\hline
\end{tabular}


reveal any significant effects of age or sort type (all $F$ 's $<1$ ), repeating the pattern of results obtained in the overall analysis. Thus, ceiling effects do not appear to account for the absence of age and sorting task differences in the correct recognition of previously-seen items.

Correct rejections. The mean number of items which were not presented in the sorting task and were correctly identified as "new" by the children may be seen in Table 4 . These correct rejections were analyzed as a function of the presentation list which each picture matched in its color and its category. A 2(color match) $\times 2$ (category match) $\times 2$ (age) $\times 2$ (sort order) analysis of variance, with age and sort order as between-subjects variables, revealed a significant color match $\times$ category match interaction $(F[1,28]$ $=5.94, p<.025$ ), but again no effects of age were found. The color match $\times$ category match interaction appears to reflect poorer performance on those distractor items which matched the same presentation list in both color and category than on those which matched different lists on the two dimensions.

\section{DISCUSSION}

Children's performance in free recall provides some support for the hypothesized developmental change in the effects of depth-of-processing on children's memory. Kindergarten children recalled more categorysorted than color-sorted items, while preschool children produced comparable recall of both types of items.

In cued recall, however, both preschool and kindergarten children recalled more category-sorted than color-sorted items. Category cueing produced higher levels of recall than did color cueing, and this advantage was greater for recall of category-sorted than color-sorted items. This finding is consistent with the encoding specificity hypothesis (Tulving \&

TABLE 4

Mean Number of Correct Rejections (Maximum = 4)

\begin{tabular}{ccc}
\hline & \multicolumn{2}{c}{ Color match } \\
\cline { 2 - 3 } & Color sort & Category sort \\
\hline Preschool & & \\
Category match: & 3.69 & 4.0 \\
$\quad$ Color sort & 3.88 & 3.63 \\
$\quad$ Category sort & & \\
Kindergarten & & 3.75 \\
Category match: & 3.75 & 3.56 \\
$\quad$ Color sort & 3.81 & \\
Category sort & &
\end{tabular}


Thomson, 1973), and suggests that the effect of cueing is not simply to raise the accessibility of all information in memory. Rather, cueing appears to elicit particular retrieval schemes whose effectiveness in accessing memory information depends on the form in which that information is encoded. However, the greater effectiveness of category than color cues, even following a color-sorting task suggests that the encoding specificity hypothesis may need some qualification. It seems likely that semantic knowledge, as well as episodically-stored information, plays a role in retrieval. That is, even if children do not encode the items in terms of category at presentation, they are likely to have some information about category membership stored in semantic memory, which could facilitate the effectiveness of category cues. In contrast, the colors of items were arbitrarily assigned in this study; hence the information necessary for utilization of color cues is only available through the retention of episodic information.

The clustering data also provide evidence that children were more able to utilize category than color information in their recall. Children showed more organization in their recall of category-sorted than color-sorted items and were more likely to cluster items by category than by color. regardless of the way in which they had been sorted at presentation.

The differing results for free as compared to cued recall for the preschool children suggest different conclusions concerning the effects of depth-ofprocessing on their memory. Since the cues provided were not uniformly effective in facilitating access to memory information, the cued recall results cannot be taken to reflect the availability of information in memory any more directly than do the free recall results. The contrast between the two sets of results points to an interaction between acquisition processes and retrieval factors, which is also indicated by the sort type $\times$ cue type interaction within the cued recall data. The precise nature of that interaction is a complex issue that is especially difficult to resolve in free recall, where little is known about the retrieval processes being used. However, it is clear that retrieval as well as encoding aspects of task performance must be considered in order to achieve an adequate understanding of the effects of depth-of-processing on young children's recall.

Recognition performance was not significantly affected by either the age level of the subjects or the type of sorting task which was performed. The absence of age differences is similar to a finding by Geis and Hall (1976), in which age differences were eliminated by controlling the encoding processes of first-, third-, and fifth-grade children. However, they obtained this result for recall, while we obtained it only for recognition. Therefore, their conclusion that encoding processes are more important than retrieval cannot be extended to the younger groups of children included in the present study. Our findings of age differences in recall but not in recognition suggest instead that retrieval factors are an important source 
of age differences in the memory performance of preschool and kindergarten children.

Effects of the sorting tasks were also eliminated through the use of a recognition procedure. This finding suggests that the different orienting tasks may not have affected the amount of information stored in memory, but only the accessibility of that information for recall. Alternatively, it is possible that storage differences did exist which simply could not be detected on the basis of recognition performance.

Comparison of the results of these three types of memory tests indicates the importance of distinguishing between voluntary and involuntary memory, and between acquisition and retrieval, in studying depth-ofprocessing in children. Since the sorting tasks controlled encoding, acquisition may be considered involuntary in these studies; however, the subsequent memory tests were always voluntary. Hence, there was an opportunity for children to utilize deliberate mnemonic strategies in retrieval. Such strategies would be particularly important in recall, where retrieval demands are greatest.

Systematic effects of age and sorting task were found in recall but not in recognition. While conclusions about the absence of depth-of-processing effects in recognition rest on acceptance of the null hypothesis, and hence cannot be made with certainty, comparison of the recognition results with those for free and cued recall suggests that retrieval factors are an important influence on the way in which different orienting tasks will affect subsequent memory performance. Likewise, although the absence of age differences in recognition cannot be conclusively accepted, it does seem clear that retrieval strategies were contributing to the age differences observed in recall. The absence of age differences on recognition tasks, and their presence on recall tasks, lends support to recent characterizations of memory development which postulate a relatively invariant structural system (Olson, 1976) and attribute age-related improvements in memory to the child's growing repertoire of voluntary strategies (Brown, 1975; Hagen et al., 1975).

\section{REFERENCES}

Appel, L. F., Cooper, R. G., McCarrel, N., Sims-Knight, J., Yussen, S. R., \& Flavell, J. H. The development of the distinction between perceiving and memorizing. Child Development, 1972, 43, 1365-1381.

Brown, A. L. Development of memory: Knowing, knowing about knowing, and knowing how to know. In H. W. Reese (Ed.), Advances in child development and behavior (Vol. 10). New York: Academic Press, 1975.

Brown, A. L., \& Murphy, M. D. Reconstruction of arbitrary versus logical sequences by preschool children. Journal of Experimental Child Psychology, 1975, 20, 307-326.

Craik, F. I. M., \& Lockhart, R. S. Levels of processing: A framework for memory research. Journal of Verbal Learning and Verbal Behavior, 1972, 11, 671-684.

Craik, F. I. M., \& Tulving, E. Depth of processing and the retention of words in episodic memory. Journal of Experimental Psychology: General, 1975, 104, 268-294. 
Flavell, J. H. Developmental studies of mediated memory. In H. W. Reese \& L. P. Lipsitt (Eds.), Advances in child development and behavior (Vol. 5). New York: Academic Press, 1970.

Flavell, J. H., Friedrichs, A. G., \& Hoyt, J. D. Developmental changes in memorization processes. Cognitive Psychology, 1970, 1, 324-340.

Geis, M. F.. \& Hall, D. M. Encoding and incidental memory in children. Journal of Experimental Child Psychology, 1976, 22, 58-66.

Hagen, J. W., Jongeward, R. H., \& Kail, R. V. Cognitive perspectives on the development of memory. In H. W. Reese (Ed.), Advances in child development and hehovior (Vol. 10). New York: Academic Press, 1975.

Hyde, T. S. \& \& Jenkins, J. J. Recall for words as a function of semantic, orthographic. and syntactic orienting tasks. Journal of Verbal Learning and Verbal Behavior. $1973,12$. $471-480$.

Keeney, T. J., Cannizzo, S. R., \& Flavell, J. H. Spontaneous and induced verbal rehearsal in a recall task. Child Development, 1967, 38, 953-966.

Loughlin, K. A., \& Daehler, M. W. The effects of distraction and added perceptual cues on the delayed reaction of very young children. Child Development, 1973, 44, 384-388.

Meacham, J. A. Soviet investigations of memory development. In R. V. Kail \& J. W. Hagen (Eds.), Perspectives on the development of memory and cognition. Hillsdale. NJ: Lawrence Erlbaum Associates, 1977.

Murphy, M. D., \& Brown, A. L. Incidental learning in preschool children as a function of level of cognitive analysis. Journal of Experimental Child Psychology, 1975, 19, 509-523.

Myers, N. A., \& Perlmutter, M. Memory in the years surrounding three. In P. A. Ornstein (Ed.), Memory development in children. Hillsdale, NJ: Lawrence Erlbaum Associates. In press.

Olson, G. M. An information processing analysis of visual memory and habituation in infants. In T. J. Tighe \& N. Leaton (Eds.), Habituation: Perspectives from child development, animal behavior, and neurophysiology. Hillsdale, NJ: Lawrence Erlbaum Associates. 1976.

Perlmutter, M., \& Myers, N. A. Recognition memory in preschool children. Developmental Psychology, 1976, 12, 271-272.

Ritter, K., Kaprove, B. H., Fitch, J. P., \& Flavell, J. H. The development of retrieval strategies in young children. Cognitive Psychology, 1973, 5, 310-321.

Smirnov, A. A. Problems of the psychology of memory. New York: Plenum Press, 1973.

Smirnov, A. A., \& Zinchenko, P. I. Problems in the psychology of memory. In M. Cole \& I. Maltzman (Eds.), Handbook of contemporary Soviet psychology. New York: Basic Books, 1969.

Tulving, E., \& Pearlstone, Z. Availability versus accessibility of information in memory for words. Journal of Verbal Learning and Verbal Behavior. 1966, 5, 381-391.

Tulving, E., \& Thomson, D. M. Encoding specificity and retrieval processes in episodic memory. Psychological Review, 1973, 80, 352-373.

Yendovitskaya, T. V. Development of memory. In A. V. Zaporozhets \& D. B. Elkonin (Eds.), Psychology of preschool children. Cambridge, MA: MIT Press, 1971.

\section{REFERENCE NOTES}

1. Murphy, M. D., Puff, C. R., \& Campione, J. C. Clustering measures and organization theory. Paper presented at SRCD, New Orleans, 1977.

2. Wingard, T. A., \& Glieberman, J. Parallel developmental changes in mnemonic and classificatory organization. Paper presented at SRCD, New Orleans, 1977.

ReCEIVED: July 5, 1977; REvised: November 10, 1977; February 2, 1978 